\title{
Premature Therapeutic Antimicrobial Treatments Can Compromise the Diagnosis of Late Periprosthetic Joint Infection
}

\author{
Alisina Shahi MD, Carl Deirmengian MD, Carlos Higuera MD, \\ Antonia Chen MD, MBA, Camilo Restrepo MD, \\ Benjamin Zmistowski MD, Javad Parvizi MD
}

Published online: 21 January 2015

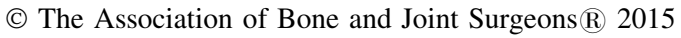

\begin{abstract}
Background In the absence of positive cultures and draining sinuses, the diagnosis of periprosthetic joint infection (PJI) relies on laboratory values. It is unknown if administration of antibiotics within 2 weeks before diagnostic evaluations can affect these tests in patients with PJI. Questions/purposes The purpose of this study was to investigate the correlation of antibiotic administration with (1) fluctuations in the synovial fluid and serology laboratory values; and (2) sensitivity of the diagnostic tests in patients with late PJI (per Musculoskeletal Infection Society [MSIS] criteria).

Methods Synovial white blood cell (WBC) count, polymorphonuclear neutrophil (PMN) percentage, and serum erythrocyte sedimentation rate (ESR) and C-reactive protein (CRP) as well as culture results were investigated in 161 patients undergoing total knee arthroplasty with late PJI diagnosed with the MSIS criteria. Depending on
\end{abstract}

One author certifies that he (JP) has or may receive payments or benefits, during the study period, in an amount of USD 10,000 to USD 100,000 , from Zimmer (Warsaw, IN, USA). One author certifies that he (JP) has or may receive payments or benefits, during the study period, in an amount of USD 10,000 to USD 100,000 from Smith \& Nephew (Andover, MA, USA). One author certifies that he (JP) has or may receive payments or benefits, during the study period, in an amount of less than USD 10,000 from ConvaTec (Skillman, NJ, USA). One author certifies that he (JP) has or may receive payments or benefits, during the study period, in an amount of USD 10,000 from TissueGene (Rockville, MD, USA). One author certifies that he (JP) has or may receive payments or benefits, during the study period, in an amount of less than USD 10,000 from CeramTec (Plochingen, Germany). One author certifies that he (JP) has or may receive payments or benefits, during the study period, in an amount of USD 10,000 from Medtronic (Minneapolis, MN, USA). One author (JP) has stock options with CD Diagnostics (Wynnewood, PA, USA), Hip Innovation Technology (Plantation, FL, USA), and PRN (Plymouth Meeting, PA, USA). whether presampling antibiotics were used, patients were divided in two groups (53 [33\%] patients were on antibiotics). The median laboratory values and the false-negative rates were compared between the two groups.

Results The median of all variables were lower in the antibiotic group compared with the other group: ESR $(\mathrm{mm} / \mathrm{hr})$ : 70 versus 85 , difference of medians $(\mathrm{DOM})=15 \mathrm{~mm} / \mathrm{hr}$, $\mathrm{p}=0.018 ; \mathrm{CRP}(\mathrm{mg} / \mathrm{L}): 72$ versus $130, \mathrm{DOM}=58 \mathrm{mg} / \mathrm{L}$, $\mathrm{p}=0.038$; synovial $\mathrm{WBC}($ cells $/ \mu \mathrm{L}): 29,170$ versus 46,900 , $\mathrm{DOM}=17,730, \mathrm{p}=0.022$; and synovial PMN\%: $88.5 \%$ versus $92.5 \%, \mathrm{DOM}=4 \%, \mathrm{p}=0.012$. Furthermore, using the MSIS cutoffs, the false-negative rates of several parameters were higher in the antibiotic group; ESR: 19.2\% (nine of 47 ) versus $6.1 \%$ (six of 99) (relative risk, 3.1; 95\% confidence interval [CI], 1.2-8.3; $\mathrm{p}=0.020)$; CRP: $14.9 \%$ (seven of 47) versus $2.00 \%$ (two of 100) (relative risk, 7.4; 95\% CI, 1.6-34.4); PMN\%: $23.1 \%$ (12 of 52) versus $9.4 \%$ (10 of 106) (relative risk, $2.4 ; 95 \% \mathrm{CI}, 1.1-5.2 ; \mathrm{p}=0.027$ ).

All ICMJE Conflict of Interest Forms for authors and Clinical Orthopaedics and Related Research ${ }^{\circledR}$ editors and board members are on file with the publication and can be viewed on request. Each author certifies that his or her institution approved the human protocol for this investigation and that all investigations were conducted in conformity with ethical principles of research.

A. Shahi, C. Deirmengian, A. Chen, C. Restrepo, B. Zmistowski, J. Parvizi $(\bowtie)$

The Rothman Institute at Thomas Jefferson University, 125 S 9th Street, Suite 1000, Philadelphia, PA 19107, USA e-mail: research@rothmaninstitute.com; parvj@aol.com

\section{Higuera}

Department of Orthopaedic Surgery, The Cleveland Clinic, Cleveland, OH, USA 
Patients in the antibiotic group also had higher rates of negative cultures: $26.4 \%$ (14 of 53) versus $12.9 \%$ (14 of 108) (relative risk, 2.0; 95\% CI, 1.05-3.9; $\mathrm{p}=0.046$ ).

Conclusions It appears that premature antibiotic treatments are associated with lower medians of diagnostic laboratory values. Thus, and in line with the guideline recommendations of the American Academy of Orthopaedic Surgeons, patients with suspected late-PJI should not receive antibiotics until the diagnosis is reached or refuted.

Level of Evidence Level III, diagnostic study.

\section{Introduction}

The diagnosis of periprosthetic joint infection (PJI) remains a challenge with no definitive standards. To address this issue, the Musculoskeletal Infection Society (MSIS) has recommended criteria to better define and diagnose PJI, criteria that underwent a modification during the 2013 International Consensus Meeting (ICM) on PJI $[18,21]$. Proper diagnosis of PJI relies on a detailed patient history, physical examinations, serologic tests, and radiologic assessments [1, 20, 22]. Additionally, isolation of the microorganism is imperative for proper diagnosis and successful treatment of the patient with PJI [18, 22]. However, the available tests for diagnosis of PJI are far from perfect. Cultures, for example, are negative in $7 \%$ to $12 \%$ of patients with PJI $[5,10,16,19]$. Culture-negative PJI can potentially complicate case classification and management and is an additional source of stress for both the patient and surgeon. Antimicrobial treatments have been reported to be a cause of culture-negative PJI $[5,17]$.

According to the definition of PJI, in the absence of major criteria, ie, communicating sinus tract or two positive cultures, serologic results are the bedrock of diagnosis [18]. Although the adverse effects of antibiotic administration on culture results has been well studied [5, 15, 26] and the clinical practice guideline of the American Academy of Orthopaedic Surgeons (AAOS) recommends withholding antibiotics for at least 2 weeks before aspiration to increase the culture yield [8], it is still unknown whether preaspiration antibiotic administration can affect synovial fluid cell counts and serology results.

The purpose of our comparative study was to evaluate the association of preaspiration antibiotic administration with (1) fluctuations in synovial fluid white blood cell (WBC) count, polymorphonuclear neutrophil (PMN) percentage, serum erythrocyte sedimentation rate (ESR), and C-reactive protein (CRP); and (2) sensitivity of the diagnostic tests in patients with PJI (per MSIS criteria) and the risk of culture-negative PJI.

\section{Materials and Methods}

After institutional review board approval, we retrospectively evaluated data in the prospective PJI databases of three institutions. Patients with late PJI after TKA, who had been treated for PJI between May 2001 and May 2013, were included for study consideration. The clinical and electronic charts were queried to determine if any of the patients had received intravenous and/or oral antibiotic within 2 weeks before initiation of investigations (joint aspiration and serologic marker measurements) for diagnosis of PJI. The following variables were then obtained for both groups - patients who did and patients who did not receive antibiotic therapy: synovial fluid WBC count, PMN percentage, serum ESR, CRP, organism profile, antibiotic type, and administration duration.

Study inclusion criteria were (1) patients who underwent TKA; and (2) developed PJI (met the MSIS diagnostic criteria for PJI [Table 1]); and (3) with preaspiration antibiotic information available in the medical records. Excluded were patients with (1) systemic inflammatory disease (such as rheumatoid arthritis [RA], systemic lupus erythematous [SLE]); (2) immunosuppressive therapy; or (3) patients with early postoperative PJI, because synovial and serologic markers are not reliable parameters for diagnosis of PJI in this setting [6, 14]. Reviewing more than 1100 infected patients in the database of the three participating centers, 182 patients were identified with a documented note about their antibiotic administration status. The patients were then categorized into two groups based on the history of antimicrobial treatments before diagnostic tests. Sixty-five patients received antibiotics, whereas 117 did not receive any antimicrobials before aspiration. Among the patients who were on antibiotics, seven patients had RA, four patients developed PJI within 30 days of their index surgery, and one was receiving sirolimus (due to a history of kidney transplant) and were therefore excluded. In the other group, patients who did not receive antibiotics, six patients underwent TKA as a result of RA and three patients developed PJI within 30 days of the index surgery and were excluded. We ended up having 161 patients with $53(33 \%)$ patients on antibiotics (Table 2).

Serum ESR, serum CRP, synovial fluid WBC count, synovial fluid neutrophil percentage, and synovial fluid culture results were recorded for each patient. The median for each test was calculated for patients with and without antibiotic treatment before testing. Additionally, the rates of false-negative results were calculated for each diagnostic test using the MSIS criteria cutoff values as a standard [18]. 
Table 1. Definition of PJI according to the ICM workgroup and threshold for the minor diagnostic criteria

PJI is present when 1 of the major criteria or 3 of 5 minor criteria exist

Major criteria

(1) 2 positive periprosthetic cultures with phenotypically identical microorganism OR

(2) A sinus tract communicating with the joint

Minor criteria

Acute PJI ( $<90$ days)

(1) Elevated serum CRP

AND

ESR
(2) Elevated SF WBC count $\underline{\mathrm{OR}}$
Changes in the leukocyte esterase strip
(3) Elevated SF PMN\%
(4) Positive histologic analysis of the periprosthetic tissue
(5) A single positive culture

\section{$100 \mathrm{mg} / \mathrm{L}$}

No threshold

10,000 cells $/ \mu \mathrm{L}$

$+\mathrm{OR}++$

$90 \%$

$>5$ neutrophil per high-power field in 5 high-power fields $(\times 400)$
Chronic PJI ( $>90$ days)

$10 \mathrm{mg} / \mathrm{L}$

$30 \mathrm{~mm} / \mathrm{hr}$

3000 cells $/ \mu \mathrm{L}$

$+\mathrm{OR}++$

$80 \%$

$>5$ neutrophil per high-power field in 5 high-power fields $(\times 400)$

$\mathrm{PJI}=$ periprosthetic joint infection; $\mathrm{ICM}=$ International Consensus Meeting; $\mathrm{CRP}=\mathrm{C}$-reactive protein; $\mathrm{ESR}=$ sedimentation rate; $\mathrm{SF}$ WBC = synovial fluid white blood cell; SF PMN = synovial fluid polymorphonuclear neutrophil.

Table 2. Patient demographics

\begin{tabular}{llll}
\hline Demographics & On antibiotics & Off antibiotics & $\mathrm{p}$ value \\
\hline Age (years) & 64.4 (range, 35-87) & 67.8 (range, 35-90) & 0.0722 \\
Sex & 25 women/28 men & 50 women/58 men & 0.9168 \\
\hline
\end{tabular}

\section{Statistical Analysis}

Given the lack of published data on the effect of antibiotics on synovial fluid laboratory values, power analysis was not performed. Descriptive statistics were used to report all laboratory values. Fisher's exact test was used to compare the rate of false-negative variables. Additionally, the Wilcoxon rank sum test was used to compare median laboratory values. A p value of $<0.05$ was considered statistically significant.

\section{Results}

Overall, patients who received preaspiration antibiotics had lower values for serologic and synovial markers for PJI. The median ESR was lower in patients who received antibiotics compared with those who did not $(70 \mathrm{~mm} / \mathrm{hr}$ versus $85 \mathrm{~mm} / \mathrm{hr}$, difference of medians $15 \mathrm{~mm} / \mathrm{hr}$; $\mathrm{p}=0.018)$ as were CRP $(72 \mathrm{mg} / \mathrm{L}$ versus $130 \mathrm{mg} / \mathrm{L}$, difference of medians $58 \mathrm{mg} / \mathrm{L} ; \mathrm{p}=0.038)$, synovial WBC $(29,170$ cells $/ \mu \mathrm{L}$ versus 46,900 cells $/ \mu \mathrm{L}$, difference of medians 17,730 cells $/ \mu \mathrm{L} ; \mathrm{p}=0.022$ ), and synovial PMN\% $(88.5 \%$ versus $92.5 \%$, difference of medians $4 \%$; $\mathrm{p}=0.012)$ (Table 3).
When using the MSIS-determined PJI thresholds for the diagnostic markers (Table 1), a larger percentage of the values was below the threshold in patients receiving antibiotics compared with patients who did not receive antibiotics (Table 4). The corresponding values for ESR were 19.2\% (nine of 47) compared with $6.1 \%$ (six of 99) (relative risk, $3.1 ; 95 \%$ confidence interval $[\mathrm{CI}], 1.2-8.3 ; \mathrm{p}=0.020)$; CRP, $14.9 \%$ (seven of 47 ) compared with $2.00 \%$ (two of 100 ) (relative risk, 7.4; 95\% CI, 1.6-34.4; $\mathrm{p}=0.005$ ); and synovial PMN percentage, $23.1 \%$ (12 of 52) compared with 9.4\% (10 of 106) (relative risk, 2.4; 95\% CI, 1.1-5.2; $\mathrm{p}=0.027)$. There was no difference in rates of false-negatives in synovial WBC count: $9.4 \%$ (five of 53) versus 6.5\% (seven of 108) (relative risk, 1.4; 95\% CI, 0.5-4.3; p = 0.53) for patients with and without antibiotics, respectively. In addition, patients with preaspiration antibiotic administration demonstrated higher rates of culture-negative infection than those without any antibiotic history (26.4\% [14 of 53] versus $12.9 \%$ [14 of 108]; relative risk, 2.0 ; 95\% CI, 1.05$3.9 ; \mathrm{p}=0.046)$ (Fig. 1).

\section{Discussion}

It is critical to rule out PJI as a possible diagnosis in patients presenting with unexplained pain or redness after 
Table 3. Comparing the median of laboratory values between the two study groups

\begin{tabular}{llllr}
\hline Parameter & $\begin{array}{l}\text { On antibiotics } \\
\text { (median) }\end{array}$ & $\begin{array}{l}\text { Off antibiotics } \\
\text { (median) }\end{array}$ & $\begin{array}{l}\text { Difference of } \\
\text { medians }\end{array}$ \\
\hline ESR $(\mathrm{mm} / \mathrm{hr})$ & 70 & 85 & 15 & 0.018 \\
CRP $(\mathrm{mg} / \mathrm{L})$ & 72 & 130 & 58 & 0.038 \\
SF WBC (cells/ $\mu \mathrm{L})$ & 29,170 & 46,900 & 17,730 & 0.022 \\
SF PMN\% & 88.5 & 92.5 & 4 & 0.012 \\
\hline
\end{tabular}

$\mathrm{ESR}=$ erythrocyte sedimentation rate; $\mathrm{CRP}=\mathrm{C}$-reactive protein; $\mathrm{SF} \mathrm{WBC}=$ synovial fluid white blood cell; $\mathrm{SF}$ PMN $=$ synovial fluid polymorphonuclear neutrophil.

Table 4. False-negative rates based on the threshold defined by the modified definition of PJI

\begin{tabular}{|c|c|c|c|c|}
\hline Parameter & $\begin{array}{l}\text { False-negative rate } \\
\text { on antibiotics }\end{array}$ & $\begin{array}{l}\text { False-negative rate } \\
\text { off antibiotics }\end{array}$ & Risk ratio $(95 \% \mathrm{CI})$ & $\mathrm{p}$ value \\
\hline ESR & $19.2 \%(9 / 47)$ & $6.1 \%(6 / 99)$ & $3.1(1.2-8.3)$ & 0.020 \\
\hline CRP & $14.9 \%(7 / 47)$ & $2.0 \%(2 / 100)$ & $7.4(1.6-34.4)$ & 0.005 \\
\hline SF WBC & $9.4 \%(5 / 53)$ & $6.5 \%(7 / 108)$ & $1.4(0.5-4.3)$ & 0.532 \\
\hline SF PMN\% & $23.1 \%(12 / 52)$ & $9.4 \%(10 / 106)$ & $2.4(1.1-5.2)$ & 0.027 \\
\hline Culture & $26.4 \%(14 / 53)$ & $12.9 \%(14 / 108)$ & $2.0(1.05-3.9)$ & 0.046 \\
\hline
\end{tabular}

$\mathrm{PJI}=$ periprosthetic joint infection; $\mathrm{ESR}=$ erythrocyte sedimentation rate; $\mathrm{CRP}=\mathrm{C}$-reactive protein; $\mathrm{SF}$ WBC $=$ synovial fluid white blood cell; SF PMN = synovial fluid polymorphonuclear neutrophil; CI = confidence interval.

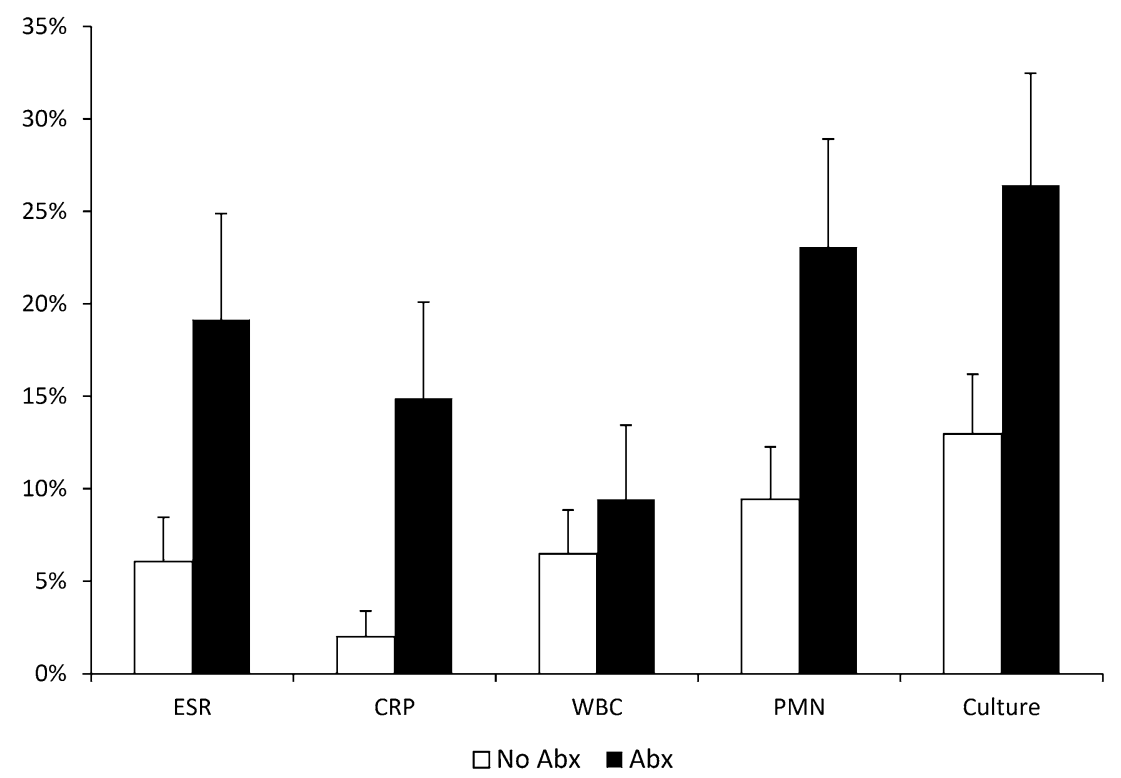

Fig. 1 Comparison of false-negative rates between patients who did receive antimicrobial treatment and patients who did not receive antimicrobial treatment before sampling for the presence of PJI is shown. Abx = antibiotics.

TKA. Given the lack of a definitive standard, MSIS created a definition for PJI, which was then modified by the ICM on PJI in 2013 [18, 21]. The MSIS diagnostic criteria are based on clinical and laboratory findings that combined can help reach the diagnosis of PJI better than any single test. Prior antimicrobial treatments have been reported to be the main cause of culture-negative PJI [5, 20, 22]; however, whether antibiotics have an effect on the serologic and other synovial parameters used for diagnosis is yet to be determined. The recommendations of the AAOS clinical practice guideline on the diagnosis of PJI for therapeutic antibiotics has mainly focused on culture yield and discouraged the use of antimicrobial treatments in patients with suspected PJI until after obtaining samples for culture. Furthermore, the guideline recommended withholding antibiotics for at least 2 weeks before intraarticular sample 
collection for culture in case the antimicrobial agents are already administered. Nevertheless, as a result of the lack of information, the guideline made no comments on any specific behavior for synovial fluid analysis when patients are on antibiotics [8]. The goal of our comparative study was to ascertain if administration of preaspiration antimicrobial influenced the value of serologic and synovial parameters associated with the diagnosis of PJI.

There are several limitations to our study and our findings should be interpreted in light of these shortcomings. The study was retrospective with all its inherent limitations such as variability in data collection and selection bias. The reason for the initiation of antimicrobial treatments in those who received antibiotics was not available. Synovial fluid cell count may vary depending on the virulence of the microorganism, duration of infection, and many other factors. The use of antimicrobial agents was not the only variable between the two groups that could have influenced the results. Therefore, to limit the confounding factors that can potentially impose an effect on inflammatory responses, patients with systematic inflammatory disease, immunosuppressant patients, and the ones who received immunosuppressive therapies were excluded from the cohort. Another limitation was that the enrolled patients were already diagnosed with PJI; according to our results, we assume that the true rate of false-negatives may in fact be even higher, because patients who received antibiotics might have been overlooked because they did not meet enough of the criteria for diagnosis. Notwithstanding these limitations, the main intention of our study was to investigate the association of antimicrobial agents with the sensitivity of the minor diagnostic criteria of PJI. Like in all other types of infection, we assumed that patients with more severe infections were more likely to receive antibiotics; therefore, even higher medians of serological and synovial tests would have been expected, but our results proved this wrong. Finally, as a result of the variation of antibiotic types, dosing, and administration duration, and the absence of detailed information for all the patients, we were unable to establish a correlation between these variables and our results.

Our results demonstrated that administration of antibiotics before investigation of patients with suspected PJI does affect the values of most synovial and serologic parameters that were used for diagnosis. There are a number of plausible mechanisms by which this phenomenon may have occurred. Reports have shown that some antibiotics can inhibit the production of interleukin (IL)-1 and tumor necrosis factor (TNF) in the lipopolysaccharidestimulated human monocyte cultures and have antiinflammatory properties. Such antiinflammatory effects are more prominent in quinolones and macrolides [2, 3, 13, 23, 24]; therefore, antimicrobial agents can decrease inflammatory responses by two mechanisms-either directly by suppressing the inflammatory cascade or indirectly by decreasing the amount of bacterial load. In addition, and because ESR and CRP are the systematic responses to inflammation, values of these inflammatory markers are likely to be decreased with the administration of antibiotics as a result of the antiinflammatory properties of some antibiotics. Cytokines are essential mediators of cell-to-cell signals in the inflammatory response. Bacterial products can activate macrophages or monocytes directly. Notwithstanding the initiating event, monocytes or macrophages often provoke the response cascade in the acute phase of inflammation. Therefore, these cells can play a decisive role in the induction of both immune and inflammatory responses [4]. When monocytes or macrophages are activated, a wide spectrum of cytokines such as IL-1, TNF, and IL-6 are released, which can induce cytokine cascade $[4,9]$. These factors establish and maintain the acute phase of inflammatory response [27]. Several studies have demonstrated that the levels of WBC and differential in the synovial fluid become elevated during PJI along with elevated blood levels. Therefore, it is believed that synovial fluid cell count and differentials follow the fluctuation patterns of the serum [11, 25, 28].

Our findings suggested that antibiotic administration is associated with higher rates of falsely negative ESR, CRP, and PMN\%. Additionally, patients who received antibiotics before sampling were more likely to have false-negative cultures; routine cultures were unable to isolate the infecting organism. This finding is in line with prior studies that demonstrated a higher rate of culture negatives in patients who received antibiotics before obtaining culture samples $[15,26]$. Based on the available evidence, the AAOS Clinical Practice Guideline for Diagnosis of PJI stated that antibiotics should not be administered to patients with suspected late PJI until the diagnosis is confirmed or refuted [8]. The Academy's guidelines recommend withholding antibiotics for at least 2 weeks before obtaining cultures from the joint. The guidelines, resulting from the lack of study evidence, do not comment on the influence of prediagnosis administration of antibiotics on synovial or serologic markers [8].

We found that patients who received antibiotics before a full evaluation for infection had been performed had lower synovial and serologic values on laboratory parameters commonly used in infection workups. If our finding represents causation and not merely association, the premature administration of antibiotics might result in a lower likelihood of diagnosing the infection in patients who have PJI. However, we distinguished between the patients in our study who received antibiotics with the intention of treating a suspected infection and those who received a single dose of preoperative antibiotics commonly used immediately 
before revision arthroplasty. Studies have shown that administration of preoperative prophylactic antibiotics has no effect on the culture yield [7]. Furthermore, studies have suggested that in proven cases of PJI, perioperative antibiotics should not be withheld, because this practice does not affect the yield of cultures obtained during revision arthroplasty [12].

Our study demonstrates that administration of antibiotics is associated with altered synovial and serologic markers used for diagnosis of PJI. It also confirms that administration of antibiotics is correlated with a higher rate of culturenegative results. Based on the findings of our study and building on the prior recommendations of the AAOS, we strongly urge the medical community to abstain from administration of therapeutic antibiotics to patients with suspected PJI before obtaining samples, because this practice does not improve the care of these patients and stands to interfere with the timely diagnosis of PJI $[5,8,17]$.

\section{References}

1. Atkins BL, Athanasou N, Deeks JJ, Crook DW, Simpson H, Peto TE, McLardy-Smith P, Berendt AR. Prospective evaluation of criteria for microbiological diagnosis of prosthetic-joint infection at revision arthroplasty. The OSIRIS Collaborative Study Group. J Clin Microbiol. 1998;36:2932-2939.

2. Bailly S, Fay M, Ferrua B, Gougerot-Pocidalo MA. Ciprofloxacin treatment in vivo increases the ex vivo capacity of lipopolysaccharide-stimulated human monocytes to produce IL-1, IL-6 and tumour necrosis factor-alpha. Clin Exp Immunol. 1991;85:331-334.

3. Bailly S, Fay M, Roche Y, Gougerot-Pocidalo MA. Effects of quinolones on tumor necrosis factor production by human monocytes. Int J Immunopharmacol. 1990;12:31-36.

4. Baumann H, Gauldie J. The acute phase response. Immunol Today. 1994;15:74-80.

5. Berbari EF, Marculescu C, Sia I, Lahr BD, Hanssen AD, Steckelberg JM, Gullerud R, Osmon DR. Culture-negative prosthetic joint infection. Clin Infect Dis. 2007;45:1113-1119.

6. Bilgen $\mathrm{O}$, Atici T, Durak K, Karaeminoğullari, Bilgen MS. $\mathrm{C}$-reactive protein values and erythrocyte sedimentation rates after total hip and total knee arthroplasty. J Int Med Res. 2001;29:7-12.

7. Burnett RSJ, Aggarwal A, Givens SA, McClure JT, Morgan PM, Barrack RL. Prophylactic antibiotics do not affect cultures in the treatment of an infected TKA: a prospective trial. Clin Orthop Relat Res. 2010;468:127-134.

8. Della Valle C, Parvizi J, Bauer TW, DiCesare PE, Evans RP, Segreti J, Spangehl M, Watters WC, Keith M, Turkelson CM, Wies JL, Sluka P, Hitchcock K, American Academy of Orthopaedic Surgeons. American Academy of Orthopaedic Surgeons clinical practice guideline on: the diagnosis of periprosthetic joint infections of the hip and knee. J Bone Joint Surg Am. 2011;93:1355-1357.

9. Durum SK, Oppenheim JJ. Proinflammatory cytokines and immunity. In: Paul WE, ed. Fundamental Immunology. 3rd ed. New York, NY, USA: Raven Press, Ltd; 1993:801-815.

10. Font-Vizcarra L, García S, Martínez-Pastor JC, Sierra JM, Soriano A. Blood culture flasks for culturing synovial fluid in prosthetic joint infections. Clin Orthop Relat Res. 2010;468: 2238-2243.
11. Ghanem E, Parvizi J, Burnett RSJ, Sharkey PF, Keshavarzi N, Aggarwal A, Barrack RL. Cell count and differential of aspirated fluid in the diagnosis of infection at the site of total knee arthroplasty. J Bone Joint Surg Am. 2008;90:1637-1643.

12. Ghanem E, Parvizi J, Clohisy J, Burnett S, Sharkey PF, Barrack R. Perioperative antibiotics should not be withheld in proven cases of periprosthetic infection. Clin Orthop Relat Res. 2007;461:44-47.

13. Iino Y, Toriyama M, Kudo K, Natori Y, Yuo A. Erythromycin inhibition of lipopolysaccharide-stimulated tumor necrosis factor alpha production by human monocytes in vitro. Ann Otol Rhinol Laryngol Suppl. 1992;157:16-20.

14. Larsson S, Thelander U, Friberg S. C-reactive protein (CRP) levels after elective orthopedic surgery. Clin Orthop Relat Res. 1992;275:237-242.

15. Malekzadeh D, Osmon DR, Lahr BD, Hanssen AD, Berbari EF. Prior use of antimicrobial therapy is a risk factor for culturenegative prosthetic joint infection. Clin Orthop Relat Res. 2010;468:2039-2045.

16. Pandey R, Berendt AR, Athanasou NA. Histological and microbiological findings in non-infected and infected revision arthroplasty tissues. The OSIRIS Collaborative Study Group. Oxford Skeletal Infection Research and Intervention Service. Arch Orthop Trauma Surg. 2000;120:570-574.

17. Parvizi J, Erkocak OF, Della Valle CJ. Culture-negative periprosthetic joint infection. J Bone Joint Surg Am. 2014;96:430-436.

18. Parvizi J, Gehrke T, International Consensus Group on Periprosthetic Joint Infection. Definition of periprosthetic joint infection. J Arthroplasty. 2014;29:1331.

19. Parvizi J, Ghanem E, Menashe S, Barrack RL, Bauer TW. Periprosthetic infection: what are the diagnostic challenges? J Bone Joint Surg Am. 2006;88(Suppl 4):138-147.

20. Parvizi J, Ghanem E, Sharkey P, Aggarwal A, Burnett RSJ, Barrack RL. Diagnosis of infected total knee: findings of a multicenter database. Clin Orthop Relat Res. 2008;466:2628-2633.

21. Parvizi J, Zmistowski B, Berbari EF, Bauer TW, Springer BD, Della Valle CJ, Garvin KL, Mont MA, Wongworawat MD, Zalavras CG. New definition for periprosthetic joint infection: from the Workgroup of the Musculoskeletal Infection Society. Clin Orthop Relat Res. 2011;469:2992-2994.

22. Patel R, Osmon DR, Hanssen AD. The diagnosis of prosthetic joint infection: current techniques and emerging technologies. Clin Orthop Relat Res. 2005;437:55-58.

23. Roche Y, Fay M, Gougerot-Pocidalo MA. Effects of quinolones on interleukin 1 production in vitro by human monocytes. Immunopharmacology. 1987;13:99-109.

24. Takeshita K, Yamagishi I, Harada M, Otomo S, Nakagawa T, Mizushima Y. Immunological and anti-inflammatory effects of clarithromycin: inhibition of interleukin 1 production of murine peritoneal macrophages. Drugs Exp Clin Res. 1989;15:527-533.

25. Trampuz A, Hanssen AD, Osmon DR, Mandrekar J, Steckelberg JM, Patel R. Synovial fluid leukocyte count and differential for the diagnosis of prosthetic knee infection. Am J Med. 2004;117:556-562.

26. Trampuz A, Piper KE, Jacobson MJ, Hanssen AD, Unni KK, Osmon DR, Mandrekar JN, Cockerill FR, Steckelberg JM, Greenleaf JF, Patel R. Sonication of removed hip and knee prostheses for diagnosis of infection. N Engl J Med. 2007;357:654-663.

27. de Waal Malefyt R, Abrams J, Bennett B, Figdor CG, de Vries JE. Interleukin 10 (IL-10) inhibits cytokine synthesis by human monocytes: an autoregulatory role of IL-10 produced by monocytes. J Exp Med. 1991;174:1209-1220.

28. Zmistowski B, Restrepo C, Huang R, Hozack WJ, Parvizi J. Periprosthetic joint infection diagnosis: a complete understanding of white blood cell count and differential. J Arthroplasty. 2012;27:1589-1593. 